Title: Prediction of CRUD deposition on PWR fuel using a state-of-theart CFD-based multi-physics computational tool

Article type: $\quad$ CFD4NRS 2014 Special Issue

Keywords: $\quad$ CRUD, CILC, high-fidelity, multi-physics CFD

Authors:

${ }^{1}$ Victor Petrov

${ }^{2}$ Brian K. Kendrick

${ }^{1}$ Daniel Walter

${ }^{1}$ Annalisa Manera

${ }^{3}$ Jeffrey Secker

Affiliations: $\quad{ }^{1}$ Department of Nuclear Engineering \& Radiological Sciences, University of Michigan, 2355 Bonisteel Boulv., Ann Arbor, MI, USA

${ }^{2}$ Theoretical Division (T-1, MS B221), Los Alamos National Laboratory, Los Alamos, New Mexico, USA, 87545

${ }^{3}$ Westinghouse Electric Company Nuclear Fuel Division 1000 Westinghouse Drive, Cranberry Township, PA, USA 16066

Corresponding author: Annalisa Manera, manera@umich.edu

Number of pages: 28

Number of figures: 16

Number of tables: 3 


\section{LIST OF FIGURES}

Fig. 1: Seabrook Cycle 5 core configuration. Fuel assemblies containing failed pins are indicated with the red boxes.

Fig. 2: Seabrook Cycle 5 Assembly G70. The 5x5 region selected for STAR-CCM+/MAMBA simulations is highlighted by the red squared region.

Fig. 3: Left: axial location of grid spacers. Right: grid spacer and adopted rod numbering. The guide tube is in position \#13.

Fig. 4: Details of the CFD mesh - pin cross section (left) and mixing vanes (right).

Fig. 5: Axial location near grid spacer \#5 for azimuthal distributions of Fig. 6.

Fig. 6: Azimuthal distribution of cladding temperature for pin \#8, near grid spacer \#5, at 4 days (top) and 502 days (bottom) respectively.

Fig. 7: 3D top view of $3 \times 3$ surrounding the center guide tube (not shown) at 502 days. The color scale indicates the CRUD thickness (in microns).

Fig. 8: $3 \times 3$ face views at 502 days.

Fig. 9: Total CRUD mass on each pin vs time

Fig. 10: 2D CRUD profiles at 502 days for pin \#8.

Fig. 11: DeCART 5x5 model showing material regions (left), and radial and azimuthal power distribution for mid-plane at 4 days (right).

Fig. 12: Azimuthal power variation for different pins at 4 days in the cycle.

Fig. 13: Left - azimuthal distribution of cladding temperature for pin \#8, near grid spacer \#5, at 502 days (dashed lines for original ANC power, solid lines for azimuthally varying ANC power distribution. Right - integral CRUD mass comparison of ANC and azimuthally varying ANC power simulations for pin 11 and 12 .

Fig. 14: 2D surface contour maps of the CRUD thickness (in microns at 502 days) for pin \#8 with and without azimuthal power variations.

Fig. 15: Normalized azimuthally averaged oxide/CRUD thickness as function of fuel height

Fig. 16: 2D contour plots of the normalized computed CRUD thickness (MAMBA) and measured oxide thickness for Seabrook fuel assembly G70 rod G09 (left) and G63 rod L07 (right). 


\section{LIST OF TABLES}

Table 1: Normalized power distribution

Table 2: Details of CFD computational mesh used for the STAR-CCM+/MAMBA CRUD simulations.

Table 3: Details of CFD computational meshes used for convergence studies $-4 \times 4$ region 


\title{
PREDICTION OF CRUD DEPOSITION ON PWR FUEL USING A STATE-OF-THE-ART CFD-BASED MULTI-PHYSICS COMPUTATIONAL TOOL
}

\author{
Victor Petrov \\ Department of Nuclear Engineering \& Radiological Sciences, University of Michigan \\ 2355 Bonisteel Boulevard, Ann Arbor, MI, USA
}

Brian K. Kendrick

Theoretical Division (T-1, MS B221), Los Alamos National Laboratory

Los Alamos, New Mexico, USA, 87545

Daniel Walter, Annalisa Manera

Department of Nuclear Engineering \& Radiological Sciences, University of Michigan 2355 Bonisteel Boulevard, Ann Arbor, MI, USA

Jeffrey Secker

Westinghouse Electric Company Nuclear Fuel Division

1000 Westinghouse Drive, Cranberry Township, PA, USA 16066

\begin{abstract}
In the present paper we report about the first attempt to demonstrate and assess the ability of state-ofthe-art high-fidelity computational tools to reproduce the complex patterns of CRUD deposits found on the surface of operating Pressurized Water Reactors (PWRs) fuel rods. A fuel assembly of the Seabrook Unit 1 PWR was selected as the test problem. During Seabrook Cycle 5, CRUD induced power shift (CIPS) and CRUD induced localized corrosion (CILC) failures were observed. Measurements of the clad oxide thickness on both failed and non-failed rods are available, together with visual observations and the results from CRUD scrapes of peripheral rods. Blind simulations were performed using the Computational Fluid Dynamics (CFD) code STAR-CCM+ coupled to an advanced chemistry code, MAMBA, developed at Los Alamos National Laboratory. The blind simulations were then compared to plant data, which were released after completion of the simulations.
\end{abstract}

\section{INTRODUCTION}

Corrosion products on fuel cladding surface have had a significant impact on reactor operation. These types of deposits are referred to as - CRUD (Chalk River Unidentified Deposit or Corrosion Residual Unidentified Deposit) and can lead to CIPS, as a consequence of the accumulation of solid boron phases on the surface of the fuel pins. CRUD can also lead to fuel failure due to CILC. The prediction of these occurrences requires a comprehensive understanding of local thermo-hydraulic and chemical processes taking place in close proximity to the cladding surface, as well as their driving factors such as power distribution and corrosion product concentration in the reactor coolant. Within the framework of a collaboration between the University of Michigan and the Los Alamos National Laboratory (LANL) under sponsorship of the DOE Consortium of Advanced Simulation of Light Water Reactor (CASL) project, the commercial CFD code STAR-CCM+ has been coupled with the macro-scale chemistry code MAMBA and to the neutron transport code DeCART in order to investigate the impact of local power and fluid dynamics parameters on the prediction of CRUD deposition. Because of the large time constant associated with the CRUD deposition rate (on the order of 0.15 microns per day), an explicit coupling between STAR-CCM+ and MAMBA was deemed sufficient. Sensitivities studies have been performed in the past in order to optimize the coupling time 
stepping. More details on the coupling scheme are given by Walter et al. (2013) and Petrov et al. (2013).

A fuel assembly from the Seabrook Unit 1 PWR Cycle 5 was selected as the test problem, since CILC failures were observed during the reactor operation. The pin power distributions during the depletion cycle were calculated by Westinghouse (WEC) using the nodal code ANC (Liu et al., 1986). A 5x5 sub-region of one of the Seabrook cycle-5 fuel assemblies was selected which included failed pins. The selected 5x5 region includes a water rod as well. This allows investigating the effect of relatively large azimuthal power variations within the pin surrounding the water rod. Details on the problem are given in section 2 and the results are discussed in section 3. In order to investigate the effects of intrapin azimuthal power distribution on CRUD deposition, additional simulations were performed using the neutron transport code DeCART, and the results are discussed in section 3.1. The simulations were performed at a time when plant data had not yet been disclosed by Westinghouse. At a later stage, the results of these blind simulations were compared to the measured plant data. The comparison with the plant data is reported in section 4.

\section{PROBLEM DESCRIPTION AND MODELING}

Seabrook Unit 1 is a 4-loop Westinghouse PWR. Compared to previous cycles, Cycle 5 consisted of a more aggressive core design, with increased cycle length and increased rod powers. At 10,000 MWD/MTU, or 260 effective full-power days (EFPD), a significant CIPS became apparent, with a maximum axial offset (AO) difference of about $-3.3 \%$.

Fuel failures started to be detected at about 350 EFPD. By end of cycle, five failed rods were observed in four distinct fuel assemblies. The assemblies containing the failed rods were part of a symmetric group of a total of twelve assemblies characterized by the highest powers for the entire cycle. All five failed rods contained Integral Fuel Burnable Absorber (IFBA) and were adjacent to guide thimbles. A sketch of the core is presented in Fig. 1. The location of the fuel assemblies containing failed fuel rods are highlighted in red.

A $5 \times 5$ region of fuel assembly G70 was selected for the analyses presented here. The fuel assembly G70 contains two failed rods in locations G9 and L7, respectively (see Fig. 2). The selected 5x5 region is highlighted in red, and contains one of the two failed rods (e.g. rod G9). The normalized power distribution for the $5 \times 5$ region is reported in Table 1 . The numbering convention adopted to identify individual fuel rods in the $5 \times 5$ region is shown in Fig. 3 (right). The notation for the azimuthal angle is also identified in the picture.

Eight grid spacers with mixing vanes are present in the fuel assembly. While all spacers have been modelled in the neutronics codes ANC and DeCART, only grid spacers \#5, \#6, and \#7 have been explicitly modelled with the CFD code. In order to limit the computational requirements (see Fig. 3, left). The axial region selected for the explicit CFD modeling of the grid spacers exhibits the highest pin powers and cladding temperatures. Therefore, it is expected that the thickest CRUD layers will be found in this region. This has been later confirmed by analysis of the plant measurements.

A three-dimensional (3D) perspective of the spacer design is presented in Fig. 3 (right). Unfortunately, at the time when the simulations were done, it was not realized that grid \#6 was rotated of $90^{\circ}$ with respect to grid \#5 and \#7. The consequences of this will be discussed in section 4.

Table 1: Normalized power distribution

\begin{tabular}{|c|c|c|c|c|}
\hline 1.354 & 1.285 & 1.401 & 1.279 & 1.341 \\
\hline 1.308 & 1.361 & 1.336 & 1.353 & 1.293 \\
\hline 1.423 & 1.339 & 0 & 1.33 & 1.405 \\
\hline 1.312 & 1.368 & 1.343 & 1.358 & 1.293 \\
\hline 1.372 & 1.309 & 1.424 & 1.299 & 1.349 \\
\hline
\end{tabular}




\subsection{CFD model}

The STAR-CCM+ computational mesh consists of over $64 \mathrm{M}$ polyhedral cells. A mesh base size of $0.4 \mathrm{~mm}$ was adopted with 4 prism layers in the proximity of the wall. The fuel solid region is modeled as well to solve for the heat conduction within the fuel. The distribution of mesh elements among coolant, fuel, cladding and guide tubes is summarized in Table 2. A cross section of the mesh near a single pin is reported in Fig. 4 (left), while a detail of the mesh of the mixing vanes is reported in Fig. 4 (right). An inlet velocity of $5.239 \mathrm{~m} / \mathrm{s}$ and pressure boundary condition is imposed for the inlet and outlet axial planes of the coolant domain, respectively. Symmetric boundary conditions are imposed on the lateral surfaces of the water domain. No-slip conditions are imposed on the grid spacers, pin walls and on the outer cladding surface. A volumetric power source is used in the fuel domain (provided by ANC or ANC/DeCART). The fluid-dynamic simulation includes conjugate heat transfer for the calculation of the temperature distribution in the fuel and cladding domains. The standard $k-\varepsilon$ turbulence model, combined with the Two-Layer All y+ wall treatment approached was employed. Past research efforts have shown that this model can predict very well the velocity field downstream of grid spacers with mixing vanes (Yan et al., 2014; OECD/NEA, 2013).

Temperature dependent materials and fluid properties were used. The convergence criteria were fixed at $10^{-6}$ for continuity, momentum, and energy. The convergence criteria in CCM+ is defined as:

$$
R_{r m s}=\sqrt{\frac{1}{n} \sum_{n} r^{2}}
$$

where $r$ is the absolute error in the solution of a particular variable and $n$ is the number of computational cells.

Table 2: Details of CFD computational mesh used for the STAR-CCM+/MAMBA CRUD simulations.

\begin{tabular}{|c|c|c|c|c|}
\hline & Fluid & Cladding & Fuel & GT \\
\hline Cells & $33,402,671$ & $13,573,982$ & $14,041,949$ & $3,081,230$ \\
\hline Faces & $161,728,592$ & $60,538,875$ & $85,524,637$ & $20,731,043$ \\
\hline Vertices & $112,379,469$ & $45,224,451$ & $70,251,624$ & $17,608,914$ \\
\hline
\end{tabular}

Table 3: Details of CFD computational meshes used for convergence studies $-4 \mathrm{x} 4$ region

\begin{tabular}{|c|c|c|c|c|}
\hline Case & $\begin{array}{c}\text { Cells } \\
\text { number* } \\
{[\mathbf{M} \text { cell }]}\end{array}$ & Base size, $\mathbf{m}$ & $\begin{array}{c}\text { Number of } \\
\text { prism layers }\end{array}$ & Mesh type \\
\hline v1 & 1.9 & $6.0 \mathrm{E}-4$ & 4 & Polyhedral \\
\hline v2 & 2.5 & $5.0 \mathrm{E}-4$ & 4 & Polyhedral \\
\hline v3 & 5.0 & $3.5 \mathrm{E}-4$ & 2 & Polyhedral \\
\hline v4 & 5.6 & $4.0 \mathrm{E}-4$ & 4 & Polyhedral \\
\hline v5 & 6.7 & $3.5 \mathrm{E}-4$ & 4 & Polyhedral \\
\hline v6 & 25.4. & $2.0 \mathrm{E}-4$ & 4 & Polyhedral \\
\hline v7 & 23.4 & $2.0 \mathrm{E}-4$ & 4 & Trimmed \\
\hline
\end{tabular}

Mesh convergence studies were performed on a $4 \times 4$ sub-region including the water rod. Six different polyhedral meshes of increasing refinement in which either the number of prism layers in the wall proximity or the base size of the computational mesh was varied (see Table 3) for more details on the mesh parameters). A seventh mesh (v7 in Table 3) was included in the study as well, characterized by the same parameters as the polyhedral finest mesh (v6), but employing trimmed elements instead of polyhedral. The different meshes were compared by analyzing relevant figures of merit such as the magnitude of the velocity field computed on cross-sections right above and below the mixing vanes of the grid spacers, and surface temperature. It was found that mesh v4 was sufficient to correctly reproduce the velocity field. Therefore, this mesh parameters were used for the modelling of the $5 \times 5$ region. Additional sensitivity studies were carried out on the influence of the turbulence modelling. The impact of turbulence modeling on the prediction of CRUD deposition is beyond the scope of the present publication and will be discussed in a future paper. 


\subsection{MAMBA model}

MAMBA (MPO Advanced Model for Boron Analysis) v2.1 simulates 3-D CRUD growth along the surface of a single fuel rod. The primary physics and chemistry associated with CRUD formation currently treated in MAMBA include: (1) solving a general non-linear, 3-D heat transport equation for the CRUD layer, including localized heat sinks due to internal boiling within the CRUD layer, (2) an adaptive grid which grows radially in time as mass deposits on the surface of the CRUD, (3) time evolving microstructure (porosity) of the CRUD layer, due to localized deposition and precipitation of nickel ferrite and lithium tetraborate within the pores of the CRUD, (4) time evolving lithium and boric acid coolant chemistry at the CRUD surface and inside the pores of the CRUD, (5) mass transport of various soluble coolant species into the interior of the CRUD, due to boiling induced Darcy flow, (6) diffusion of various soluble species inside the CRUD, due to the flow induced concentration gradients within the CRUD layer, and (7) mass evaporation in the form of steam vapor, due to the localized boiling inside the CRUD layer.

MAMBA's 3D cylindrical grid consisted of 148 axial nodes with a uniform spacing of $2.5 \mathrm{~cm}$. The azimuthal grid consisted of 16 azimuthal nodes with a uniform spacing of $22.5^{\circ}$. The radial grid is an adaptive time dependent grid which varies as a function of the local CRUD thickness. A uniform radial spacing of $5 \mu \mathrm{m}$ was used with $\mathrm{r}(0)=0$ corresponding to the cladding surface. A thin "seed" layer $(5 \mu \mathrm{m})$ of CRUD was initialized along the entire 2D surface of the rod at time zero. As for the case of the CFD computational mesh, the MAMBA nodalization parameters were selected on the basis of appropriate mesh convergence studies.

The measured time-dependent boron and lithium coolant concentrations for Seabrook Cycle 5 were used as input for the MAMBA code. The soluble and particulate nickel concentrations were also estimated and input to MAMBA. A constant average value of $32 \mathrm{~cm}^{3} / \mathrm{kg}$ (STP) was used for the dissolved hydrogen concentration, according to Westinghouse specifications. These concentrations are used by MAMBA's internal thermodynamics models to compute the local $\mathrm{pH}$ and solubilities of various species of interest, such as: boric acid, lithium tetraborate, lithium monoborate, bonaccordite, nickel metal, nickel oxide and nickel ferrite. In this work, the solid skeleton of the CRUD layer was assumed to consist of only nickel ferrite (due to surface deposition of the particulates suspended in the coolant). The particulate surface deposition rate is governed by two temperature dependent Arrhenius rate coefficients: one for boiling and another for non-boiling regions. The boiling and non-boiling CRUD deposition rates were determined in previous studies (Walter et al., 2013; Petrov et al., 2013). Depending upon the local internal CRUD temperature, the soluble $\mathrm{Ni}$ and boric acid species in the liquid within the pores of the CRUD can deposit and precipitate increasing the density of the CRUD layer especially near the cladding surface. The pore fill rate due to the continual slow deposition of $\mathrm{Ni}$ phases within the pores of the CRUD was also determined in a previous study by comparing the MAMBA computed CRUD density with the experimental data from the Westinghouse WALT loop (Kendrick and Barber, 2012).

The STAR-CCM+ computed surface heat flux was used to set the MAMBA temperature boundary condition at the cladding surface $(\mathrm{r}=0)$ and the STAR-CCM+ computed CRUD/coolant surface temperature was used to set the MAMBA CRUD/coolant temperature boundary condition. The 3D temperature distribution within the CRUD layer was computed by iteratively solving a general 3D non-linear heat transport equation with internal "sinks" due to chimney boiling. The effective bulk CRUD thermal conductivity is a function of the local CRUD properties such as temperature and porosity $(\varepsilon)$ and is computed using a mixing fraction: $\mathrm{k}_{\mathrm{eff}}=(1-\varepsilon) \mathrm{k}_{\mathrm{s}}+\varepsilon \mathrm{k}_{\mathrm{w}}$ where $\mathrm{k}_{\mathrm{s}}$ and $\mathrm{k}_{\mathrm{w}}(\mathrm{T})$ are the thermal conductivities of solid CRUD and water, respectively. A similar expression is used to compute the CRUD's effective bulk specific heat capacity. The chimney boiling model is a modified Cohen type model (Cohen, 1974; Henshaw et al., 2006) where the bulk heat power density leaving a given CRUD volume element is expressed as $q=H_{c} N_{c} A_{c} \kappa\left(T-T_{s a t}\right)$ where $H_{c}$ is an effective bulk chimney heat transfer coefficient, $N_{c}$ is the chimney surface density, $A_{c}$ is an effective bulk chimney boiling surface area (which is a function of the chimney radius), $\kappa$ is a porosity dependent CRUD permeability, $\mathrm{T}$ is the local CRUD temperature, and $\mathrm{T}_{\text {sat }}$ is the local liquid saturation temperature (which is a function of the local soluble boric acid concentration). The chimney parameters and the CRUD thermal conductivity $\mathrm{k}_{\mathrm{s}}$ were previously optimized by fitting the MAMBA computed CRUD 
temperature to the experimental WEC WALT loop data (Kendrick and Barber, 2012). These values are discussed in previous publications (Walter et al., 2013; Petrov et al., 2013) and were kept unchanged in the present work. We note that if $\mathrm{T}<\mathrm{T}_{\text {sat }}$ then the local $\mathrm{q}$ is set equal to zero (i.e., there is no internal chimney boiling in that CRUD volume element). The boiling heat flux q given above acts as a localized "sink" in the heat transport calculation and it is also used to compute the radial flow velocity of the coolant into the CRUD layer. The radial flow replenishes and concentrates the various soluble species within the pores of the CRUD (i.e., boric acid and Ni). The concentrations of these species can increase significantly near the cladding surface if the advective flux of the soluble species due to boiling induced flow is significantly greater than the concentration gradient induced diffusive flux in the opposite direction. Both the radial flow and diffusion are included in MAMBA's internal mass transport calculations. These mechanisms contribute to the precipitation of the boron species within the pores of the CRUD and also to the densification of the CRUD near the cladding surface (due to the slow Ni deposition inside the pores). The thermal resistance and thickness of the CRUD layer are computed by MAMBA. These data are then passed to STAR-CCM+ at each depletion step to update its internal conjugate heat transfer calculation (which accounts for the temperature drop across the CRUD layer). We note that in boiling regions the heat removed due to boiling reduces the temperature drop across the CRUD layer. The smaller $\Delta \mathrm{T}$ results in a smaller effective thermal resistance. In addition, the turbulent kinetic energy computed by STAR-CCM+ is used by MAMBA at each depletion step to estimate the CRUD's surface erosion rate.

\subsection{Pin-by-pin power distribution}

The pin by pin power distribution was computed by Westinghouse using the ANC 3D nodal code. This code is based on the neutron diffusion approximation and utilizes a pin power reconstruction method to compute pin power distributions. However, ANC does not provide azimuthal (or radial) power variations within the pin. The 3D power distribution for the Seabrook core Cycle 5 was computed using ANC at different burnup stages during the cycle and input in STAR-CCM+ as volumetric heat source in the fuel region. The relative pin-by-pin power distribution at the beginning of Seabrook cycle 5 for the $5 \times 5$ region of interest is reported in Table 1.

\subsection{Run time}

The CFD calculations were performed on 62 cores (IBM x3755 M3, with 4 CPU AMD Opteron 6282 SE), with a run time of 11.23 days. The MAMBA simulations were run on 24 cores (Intel Xenon 2670), with a total run time of 4.85 hours, data processing took a total of 19.68 hours.

\section{RESULTS}

The azimuthal temperature distributions for pin \#8 at three different elevations in the proximity of spacer \#5 (see Fig. 3) are reported in Fig. 6 at days 4 and 502 respectively. The locations of the three elevations are illustrated in Fig. 5, with rim 1 being upstream and rim 3 downstream of the mixing vanes respectively. Upstream of the mixing vanes, the azimuthal variations of the cladding temperature are due to the different amount of coolant that is surrounding the pin surface which is governed by the subchannel geometry. In contrast, downstream of the mixing vanes (rim 3), the temperature azimuthal variations are significantly amplified by the flow swirl induced by the vanes. The flow swirl causes also a phase shift in the azimuthal temperature distribution.

The effect of the CRUD induced thermal resistance is clearly visible when comparing the temperature values at 4 and 502 days respectively. At 502 days, a considerable amount of CRUD has deposited on the rod surface, as shown in Fig. 7 (right). The patterns of the CRUD deposits in the simulation discussed here are solely caused by the flow swirls induced by the grid spacer mixing vanes, since no azimuthal power variation has been considered here, as discussed in chapter 2.3. A significant variation of the turbulent kinetic energy and shear stresses are observed in proximity of the fuel cladding wall. As expected, this is strongly dependent on the orientation of the mixing vanes.

It is found that the CRUD deposits are out-of-phase with the flow swirl since regions of high turbulent kinetic energy (or shear stress) correspond to higher heat transfer coefficients (cooler cladding surface) and higher CRUD erosion rates (Petrov et al., 2013). It is noteworthy that, because of the particular 
orientation of the mixing vanes, rod \#8 exhibits high CRUD deposit thickness on the surface not facing the water rod, as can be seen in Fig. 7 (right).

Three-dimensional perspective plots of the eight rods surrounding the guide tube are plotted in Fig. 7 and Fig. 8. The swirling CRUD patterns, mixing vane induced erosion effects, and $180^{\circ}$ symmetry are clearly visible in the upper spans. In Fig. 8, the locations of the spacer grids \#5, 6, and 7 are labelled by arrows. Due to the $180^{\circ}$ symmetry, the front face of rod \#7 is the same as the back face of rod \#19 and vice versa (see sketch in Fig. 7, left). Similar front/back symmetry holds for the other symmetric partners: $(8,18),(9,17)$, and $(12,14)$.

Fig. 9 plots the total CRUD mass on each rod integrated over the 3D MAMBA grid. A small jump in the mass accumulation rate is observed at 50 days due to a temporary spike in the Seabrook coolant Ni concentration induced by a reactor trip and subsequent extended operation at reduced power. The different coloured curves correspond to the eight rods of the $3 \times 3$ region surrounding the guide tube (see red square in Fig. 7, left) and the yellow curves correspond to the other rods of the $5 \times 5$ subassembly. Rods \#12 (red) and 14 (blue) accumulate the most CRUD mass by the end of the cycle at 502 days.

In agreement with plant data, there is no correlation for CRUD deposits preferred orientation with the location of the water rods, as the highest CRUD thicknesses are not necessarily observed on the rods faces adjacent to the water rods (see Fig. 7). Note that although rod \#8 is facing the water rod, it exhibits maximum CRUD thickness on the side not facing the water rod. Also, Rod \#8 has a slightly higher power than rod \#12 (see Table 1), despite the CRUD mass accumulated on rod \#12 is higher. This shows that localized thermal hydraulic effects such as the one induced by mixing vane orientations play an important role in determining both the amount of CRUD that deposits on each pin and the location where the deposits occur. The maximum CRUD thickness at the end of the cycle varied between 70-80 microns. The CRUD thickness for rods \#12, 14, 8, and 18 within the central 3x3 region around the guide tube reached a maximum of 75 microns. These CRUD thickness values are consistent with the experimental data from the Seabrook CRUD scrapes (Secker, 2011). Very little CRUD (i.e, thickness < 10 microns) is deposited below grid \#5 where the cladding surface temperature is lower and very little if any subcooled nucleate boiling (SNB) occurs (i.e. the cladding surface temperature is below the coolant's saturation temperature Tsat). Significant CRUD deposits occur above grid \#5 with the largest amount of CRUD being deposited within span \#6 (i.e., between grids \#6 and 7). This trend is consistent with the Seabrook CRUD data. The CRUD deposits track the coolant flow patterns and are thickest where the cladding surface is hottest and SNB occurs. In contrast, very little CRUD deposits in the vicinity of the mixing vanes where the cladding surface temperature is lower and significant surface erosion occurs due to the large turbulent kinetic energy (or shear stresses) in these regions.

The four 2D profile plots in Fig. 10 for rod \#8 show the CRUD's porosity, temperature, boiling heat flux, and boron concentration as a function of the axial height $\mathrm{z}$ and the radial distance from the cladding surface $r$. By the end of Seabrook cycle 5, the density of the CRUD layer near the cladding surface has increased (i.e. the porosity has decreased from 0.7 (red) to 0.42 (yellow) which is consistent with the observations from Seabrook CRUD scrapes. The maximum CRUD thickness in span \#6 for both of these pins is 75 microns and is also consistent with the Seabrook CRUD scrape data. The three "dips" in the CRUD's thickness at axial locations around 222, 274, and $326 \mathrm{~cm}$ correspond to the location of the three spacer grids and are due to the reduced cladding surface temperature and surface erosion effects from the mixing vanes. As expected, the CRUD temperature is hottest (red) in the denser regions near the cladding surface reaching a maximum temperature of approximately $348^{\circ} \mathrm{C}$. The heat flux density due to chimney boiling within the CRUD layer acts as a local heat sink and also induces radial flow of liquid from the surface of the CRUD down into the CRUD layer. Significant boiling within the CRUD layer is indicated by the light-blue colored regions. The largest boiling (red) occurs near the surface of the CRUD. The boiling within the CRUD layer also enhances the CRUD's deposition rate leading to thicker CRUD in boiling regions. Soluble boric acid species concentrate near the cladding surface (red) due to the boiling induced flow. 
As the local boric acid concentration increases, it can eventually exceed the solubility limit for a solid phase boron compound such as lithium tetraborate $\left(\mathrm{Li}_{2} \mathrm{~B}_{4} \mathrm{O}_{7}\right)$. Once the solubility limit is exceeded, the precipitation of the solid phase occurs which quickly fills a significant fraction of the pores while simultaneously shutting off the boiling induced flow in that region.

\subsection{Pin-by-pin power distribution}

The ANC pin power distributions were adjusted to include azimuthal variation (circumferentially around the rod) in order to investigate power azimuthal variations on CRUD deposits. The normalized azimuthal variation of the power was computed using the neutron transport MOC (Method of Characteristics) code DeCART. The sub-pin level power distribution computed by DeCART includes radial, azimuthal, and axial dependence. The axial discretization was the same as in the ANC model, and the radial and azimuthal discretization for a representative plane is shown in Fig. 11. The azimuthal variation of the power is clearly demonstrated in the pins surrounding the guide thimble (i.e. water rod). Five equal volume radial regions exist within each fuel cell and 16 azimuthal regions are defined in each pin cell. To extract the azimuthal power distribution for a given plane within a given pin, the radial regions belonging to each azimuthal region were averaged. Then, the 16 azimuthal power values were normalized, and the ANC power distributions were adjusted, while ensuring the total power was preserved in each planar segment for all pins.

The normalized variation in the azimuthal power for the four pins surrounding the guide tube is shown in Fig. 12. Variations in the azimuthal power approach 3\% for the side of the pins that face the guide tube. This increase in power occurs due to the additional water present within the guide tube, which increases local neutron moderation.

The impact of the power azimuthal variation on the cladding temperature can be better observed in Fig. 13 (left). Here the results of the azimuthal variation of the cladding temperature obtained for pin \#8 in the proximity of spacer \#5 are shown for the base case with no azimuthal power variations (dashed lines) and the case with azimuthal power variations (solid lines). As expected, the larger impacts of the power variation occurs around $90^{\circ}$, where pin $\# 8$ is facing the water rod. However, at this location the azimuthal power variation accounts for an increase of the cladding temperature of $2 \mathrm{~K}$ only, while the peak-to-peak azimuthal temperature variations induced by changes in the cooling characteristics (size of subchannel or flow-swirl induced by mixing vanes) are above $15 \mathrm{~K}$. It can therefore be concluded that for CRUD-related simulations, the fidelity of the neutronics modeling is not as essential as the fidelity of the thermal-hydraulics modeling. A CFD simulation coupled with a nodal diffusion code such as ANC may be sufficient to achieve the necessary level of accuracy.

The effect of the azimuthal power variations on the CRUD deposits can be seen in Fig. 13 (right) and Fig. 14. In Fig. 13 (right) the total mass of CRUD deposited on pins \#11 and \#12 is reported as a function of the depletion cycle with and without azimuthal power variations respectively. In Fig. 14 the contour plots of the CRUD thickness at 502 days are reported for pin \#8 as function of azimuthal and axial position. Small differences are observed between the two cases with and without intra-pin azimuthal power variations. The most notable effect is an increased axial extension of the CRUD in Fig. 14 in the region near $\theta=270^{\circ}$ and a decreased axial extent near $\theta=100^{\circ}$ when azimuthal power variations are taken into account. These small differences confirm that the primary effect on azimuthal CRUD deposition patterns along the surface of the rod are due to the thermal-hydraulics variations associated with the complex fluid flow patterns. However, additional studies using a fully coupled neutronics/CFD/CRUD framework are needed to confirm this preliminary finding. Larger azimuthal power variations could occur in a fully coupled simulation due to feedback effects between the CFD and neutronics.

\section{COMPARISON WITH PLANT DATA}

Measurements of the oxide thickness were performed on several failed and non-failed fuel rods from Seabrook cycle 5 (Secker, 2011). These measurements were performed along the axial length of the rod and were repeated at several azimuthal locations. Azimuthal scans around the rods at a few selected axial heights were also performed. However, the crud analysis is mostly qualitative and is 
based primarily on visual examinations of the various faces of the rods in the more thickly cruded upper spans 5, 6 and 7. In this initial comparison to the Seabrook data, we will focus on the failed rod G09 in FA G70 and on selected rods from G70's symmetric partner G63, namely rods G09 and L07. We compare the measured oxide thickness with MAMBA's computed crud thickness under the assumption that the thicker oxide layers (> 75 microns) are most likely due to the presence of crud (i.e., CILC). Because there are no quantitative data on the CRUD thickness, only a qualitative comparison can be made.

As mentioned previously, the orientations for spacer grids 5 and 7 are different than those for grid 6 , implying that the coolant flow patterns past grid \#5 and \#7 will be different than the flow past grid \#6, which can lead to differences in crud deposition patterns on the surfaces of the rods. Even though our present simulation assumed the same mixing vane orientation for all three grids, we can make initial comparisons to the Seabrook data by using the results from our simulation on two different rods which have the appropriate mixing vane orientation. Specifically, the vane orientation around our rod 8 corresponds to the Seabrook orientation on grid spans 5 and 7 for rod G09 in fuel assembly G70. The vane orientation around our rod 12 (rotated by $90^{\circ}$ into the rod 8 position) corresponds the Seabrook orientation on grid span 6 for rod G09.

The computed azimuthally averaged CRUD thickness as function of fuel height is reported in Fig. 15 (left) for rods \#8 and \#12 together with the measured oxide thickness. The oxide thickness does appear to correlate with the crud thickness. In particular, the dips in oxide thickness correspond to the top of the spacer grids where the turbulent flow past the mixing vanes suppresses crud formation. The oxide and CRUD thickness are both largest in the hotter span 6 and decrease rapidly in span 7, where the heat flux is lower. Consistent with plant data, no significant deposits are observed at elevations below $200 \mathrm{~cm}$. In Fig. 15 (bottom) the comparison is done for the rod G09 of fuel assembly G63 (in this case the simulation pin \#8 is the corresponding pin). The measured oxide thickness on this rod is only available at an angle $\theta_{\mathrm{m}}=0^{\circ}$ (corresponding to $315^{\circ}$ in the computation coordinate system). The MAMBA computed CRUD thickness on rod \#8 at $\theta=315^{\circ}$ and 502 days is superimposed (blue). The correlation between the measured oxide thickness and the MAMBA computed CRUD thickness is excellent. The only discrepancy is for the CRUD deposition below grid 5. However, since grid 4 was not included in the CFD model, the effect of CRUD erosion on span 4 was not modelled in the simulations. Downstream of the modelled grid spacers (spans 5 to 7 ) the agreement is very good. In agreement with plant data, no significant CRUD deposits are observed in span 7. Again, the sharp dips in both the measured oxide and CRUD thickness correspond to the tops of the spacer grids where the turbulent flow past the mixing vanes impedes crud formation. In Fig. 16 the 2D contour plots of the normalized computed CRUD thickness is plotted together with the normalized measured oxide thickness. Because of the error in the orientation of spacer grid \#6, only span 6 can be compared for pin \#12 and only spans 5 and 7 can be compared for pin \#8. In both cases, for the regions where the comparison is meaningful, the location where MAMBA predicts the thickest CRUD layer corresponds very well with the locations where the highest oxide layer have been observed in the measured data, especially considering that the measured data available for the counterplots in Fig. 16 were limited to four angles for rod G63L7 and eight angles for rod G70_G09, respectively (the MAMBA nodalization consisted of 16 azimuthal nodes instead).

\section{CONCLUSIONS}

The coupled STAR-CCM+/MAMBA code has been found to accurately predict the pattern of CRUD deposits and the locations of maximum CRUD thickness. In accordance with experimental data, thickest CRUD deposits are not necessarily found in correspondence of highest pin powers, the dominant effect being the flow swirl induced by the mixing vanes. In this respect, it has been concluded that in order to correctly predict the pattern of the CRUD deposits, an accurate representation of the mixing vanes geometry and orientation is crucial. It has also been found that the mass evaporation flux and thick CRUD are directly correlated. Therefore, computed mass evaporation flux maps can be used to help optimize spacer grids and mixing vane orientation designs to minimize CRUD. 
The results presented in this work represents the first detailed comparison of a high-fidelity two-way coupled CFD / crud chemistry modeling approach to a realistic and well documented plant cycle. The obtained results are encouraging, especially considering that no parameter optimizations were performed, and the calculations were completed before the plant data became available. All of the MAMBA model parameters were fixed in this study, being based on previous WALT Loop validation studies (Kendrick and Barber, 2012) and general plant observations (Walter et al., 2013; Petrov et al., 2013).

\section{ACKNOWLEDGMENTS}

This work was partially supported by the NRC faculty development grant NRC-HQ-11-G-38-0038, and the Consortium for Advanced Simulation of Light Water Reactors (CASL) under U.S. Department of Energy Contract No. DE-AC05-00OR22725. 


\section{REFERENCES}

Cohen, P., "Heat and mass transfer for boiling in porous deposits with chimneys". AIChE Symposium Series, 70 (138), 1974.

Henshaw, J., et al., "A model of chemistry and thermal hydraulics in PWR fuel crud deposits", Journal of Nuclear Materials 353, 1-11, 2006.

Kendrick, B.K., Barber, J., "Initial validation and benchmark study of 3D MAMBA v2.0 against the WALT loop experiment and BOA v3.0". CASL report MPOCRUD.P5.02, 2012.

Liu Y., et al, Pin Power Prediction in the Westinghouse Advanced Nodal Code, 1986, Trans. Am. Nuclear Soc., Vol. 53, pp 246.

OECD/NEA, "Report of the OECD/NEA-KAERI Rod Bundle CFD Benchmark Exercise", July 2013, NEA/CSNI/R(2013)5.

Petrov, V., Kendrick, B.K., Walter, D., Manera, A., "Impact of fluid-dynamic 3D spatial effects on the prediction of CRUD deposition in a 4x4 PWR sub-assembly", Proc. of NURETH-15, Pisa, Italy, 12 16 May 2013.

Secker, J., "AMA.CHALNG.07.Y1 Test Problem and Validation Data Package for CRUD", DOE/CASL deliverable, 2011.

Yan, J., Yun, P., Smith, L.D., Karoutas, Z.E., Jofrre, P., "PWR Fuel Sub-Channel Thermal Mixing CFD Model Development and Validation", Proc. of CFD4NRS-5, Zurich, Switzerland, 9 - 11 September 2014.

Walter, D., et al., "High-fidelity simulation of crud deposition on a fuel pin with grid spacers - a proofof-principle using the fully coupled MAMBA/DeCART/STAR-CCM+ code", Proc. of NURETH-15, Pisa, Italy, 12 - 16 May 2013. 


\begin{tabular}{|c|c|c|c|c|c|c|c|c|c|c|c|c|c|c|c|c|}
\hline $\mathrm{R}$ & $\mathrm{P}$ & $\mathrm{N}$ & $M$ & $\mathrm{~L}$ & K & $J$ & $\mathrm{H}$ & $\mathrm{G}$ & $\mathrm{F}$ & $E$ & $D$ & C & $B$ & $A$ & & \\
\hline & & & & E45 & $F 11$ & $F 26$ & E41 & F13 & $F 33$ & E48 & & & & & 1 & \\
\hline & & $\mathrm{E} 65$ & $\mathrm{~F} 10$ & G25 & G33 & G34 & $F 51$ & G35 & G36 & G26 & F34 & $\mathrm{E} 68$ & & & 2 & \\
\hline & E70 & G37 & G38 & G61 & $F 40$ & F59 & G01 & F70 & $F 38$ & G62 & G39 & G40 & E53 & & 3 & \\
\hline & $\mathrm{F} 20$ & G41 & $F 45$ & $\mathrm{~F} 60$ & E01 & G73 & $\mathrm{F} 03$ & G74 & E07 & $F 72$ & F44 & G42 & $F 22$ & & 4 & \\
\hline E49 & G27 & $\begin{array}{l}\text { G63 } \\
\text { Failed }\end{array}$ & $\mathrm{F} 63$ & $\mathrm{~F} 53$ & G02 & E75 & $\begin{array}{l}\text { G64 } \\
\text { Failed }\end{array}$ & E56 & G03 & $F 52$ & F57 & G65 & G28 & E43 & 5 & Failures \\
\hline F19 & G43 & $\mathrm{F} 48$ & E03 & G04 & F27 & G05 & $\mathrm{F} 30$ & 606 & $F 08$ & G07 & E08 & F 39 & G44 & $\mathrm{F} 23$ & 6 & G/U Rod Gy \\
\hline $\mathrm{F} 29$ & G45 & $\mathrm{F} 71$ & G75 & E62 & G08 & E61 & 609 & $\mathrm{E} 73$ & G10 & E54 & G76 & $\mathrm{F} 58$ & G46 & F18 & 7 & G64 Rod E12 \\
\hline E51 & F54 & G11 & F01 & G66 & $\mathrm{F} 32$ & G12 & A04 & G13 & F07 & G67 & $\mathrm{F} 04$ & G14 & F55 & E46 & 8 & G63 Rod K12 \\
\hline $\mathrm{F} 14$ & G47 & F65 & G77 & E74 & G15 & $E 72$ & G16 & E63 & G17 & E76 & G78 & F66 & G48 & F17 & 9 & G69 Rod M14 \\
\hline$F 24$ & G49 & F47 & E04 & G18 & $F 28$ & G19 & F09 & G20 & F15 & G21 & E02 & F37 & G50 & $F_{36}$ & 10 & \\
\hline E44 & G29 & G68 & $F 62$ & $F 56$ & G22 & E58 & $\begin{array}{l}\text { G69 } \\
\text { Failed }\end{array}$ & E57 & G23 & $F 49$ & F64 & $\begin{array}{l}\text { G70 } \\
\text { Failed }\end{array}$ & G30 & E39 & 11 & \\
\hline & $\mathrm{F} 21$ & G51 & $F 46$ & $F 68$ & E06 & G79 & $\mathrm{F} 02$ & G80 & E05 & F67 & $\mathrm{F} 41$ & G52 & $F 25$ & & 12 & \\
\hline & E67 & G53 & G54 & G71 & $\mathrm{F} 43$ & $F 61$ & G24 & $\mathrm{F} 69$ & $\mathrm{~F} 42$ & G72 & G55 & G56 & E66 & & 13 & \\
\hline & & $\mathrm{E} 60$ & F06 & G31 & G57 & G58 & $\mathrm{F} 50$ & G59 & G60 & G32 & F 35 & E55 & & & 14 & \\
\hline & & & & E52 & $\mathrm{F} 16$ & $F_{31}$ & E50 & $\mathrm{F} 12$ & F05 & E38 & & & & & 15 & \\
\hline
\end{tabular}

Fig. 1: Seabrook Cycle 5 core configuration. Fuel assemblies containing failed pins are indicated with the red boxes. 
$\square$ = location of guide tubes

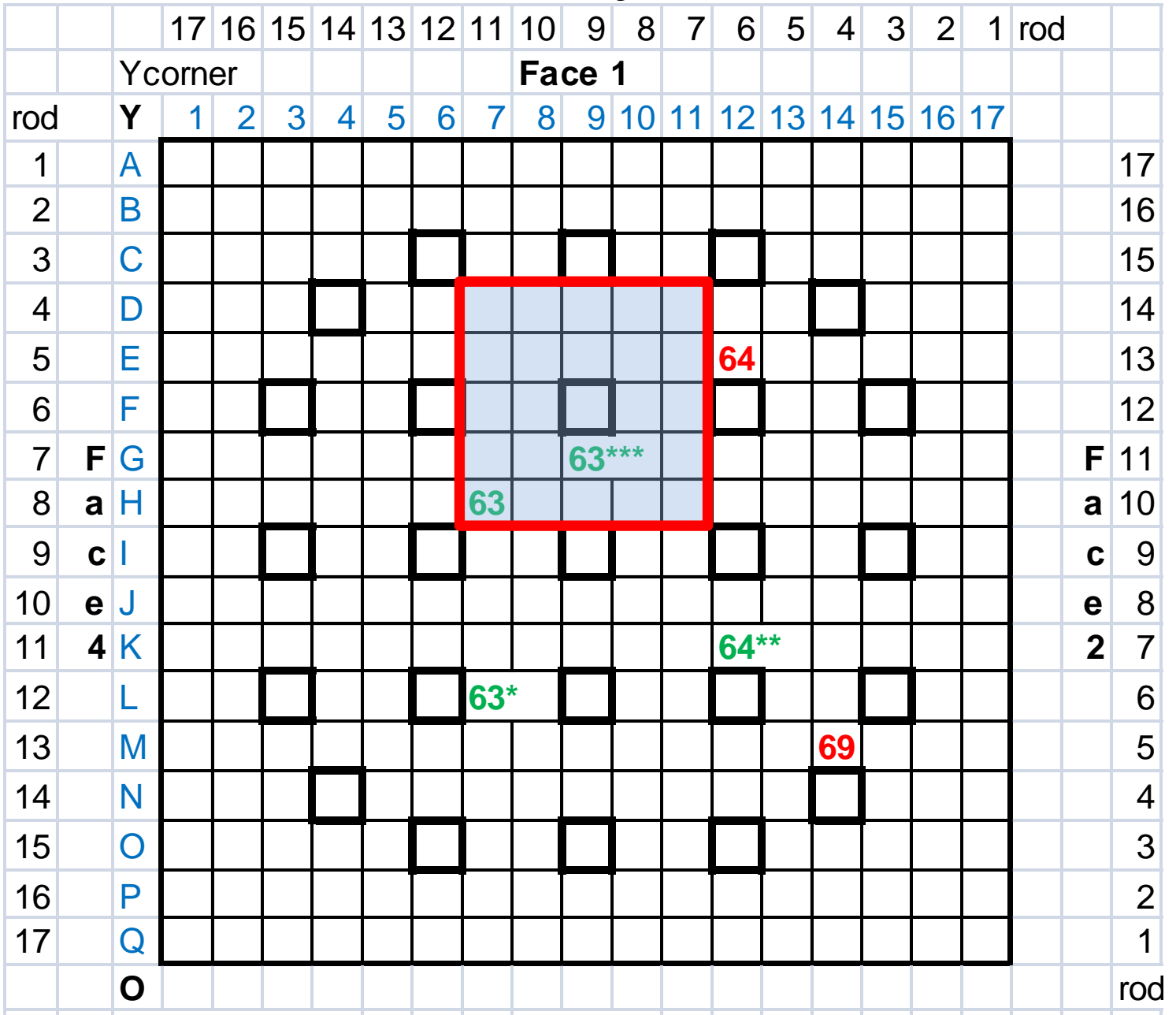

\begin{tabular}{|l|l|l|l|l|l} 
Reference Hole & Face 3 \\
\hline
\end{tabular}

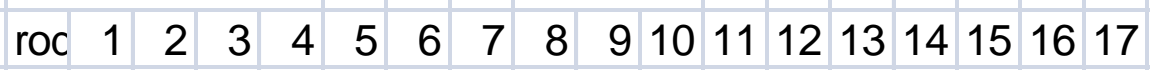

Fig. 2: Seabrook Cycle 5 Assembly G70. The $5 \times 5$ region selected for STAR-CCM+/MAMBA simulations is highlighted by the red squared region. 

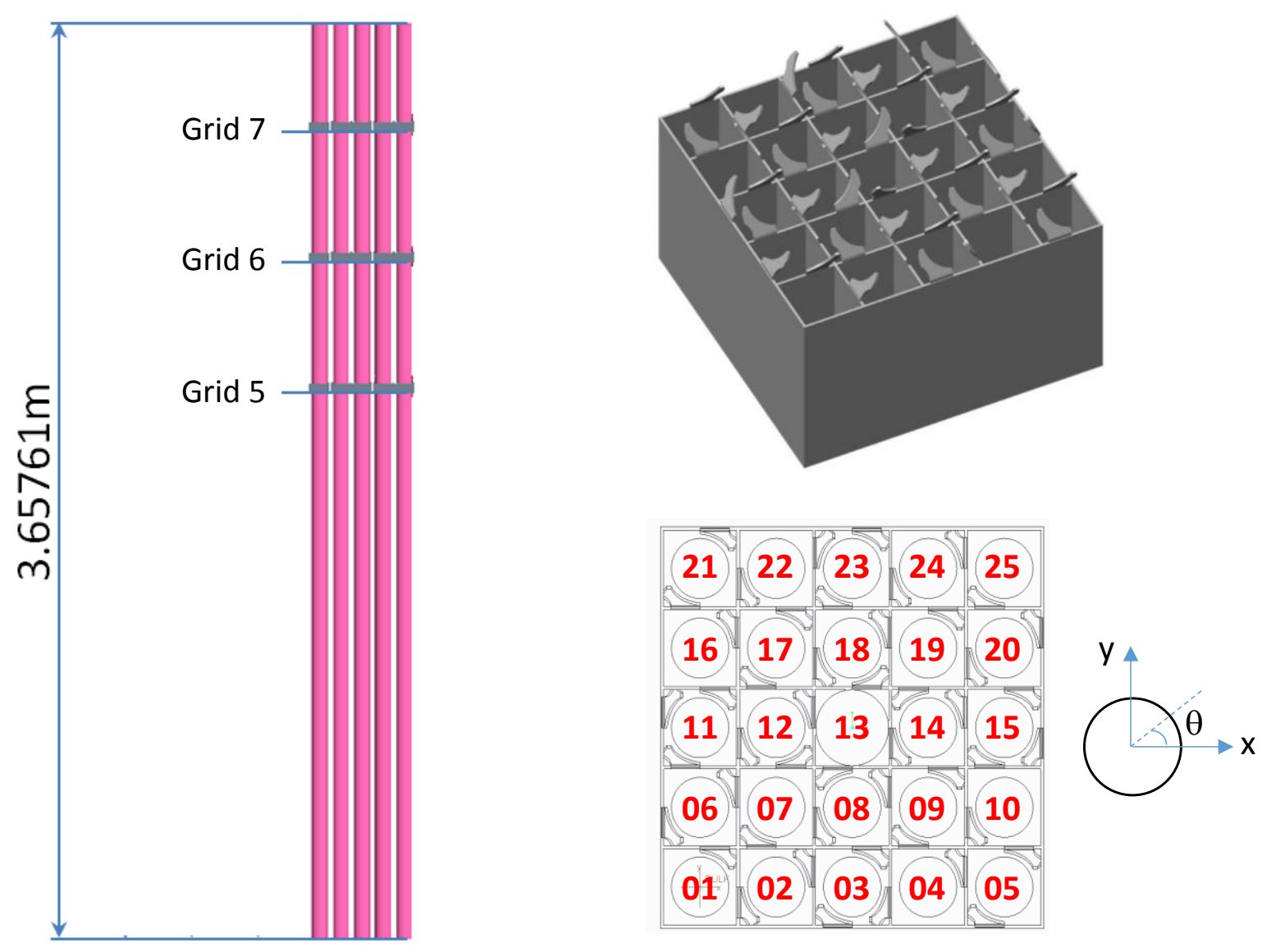

Fig. 3: Left: axial location of grid spacers. Right: grid spacer and adopted rod numbering. The guide tube is in position \#13. 

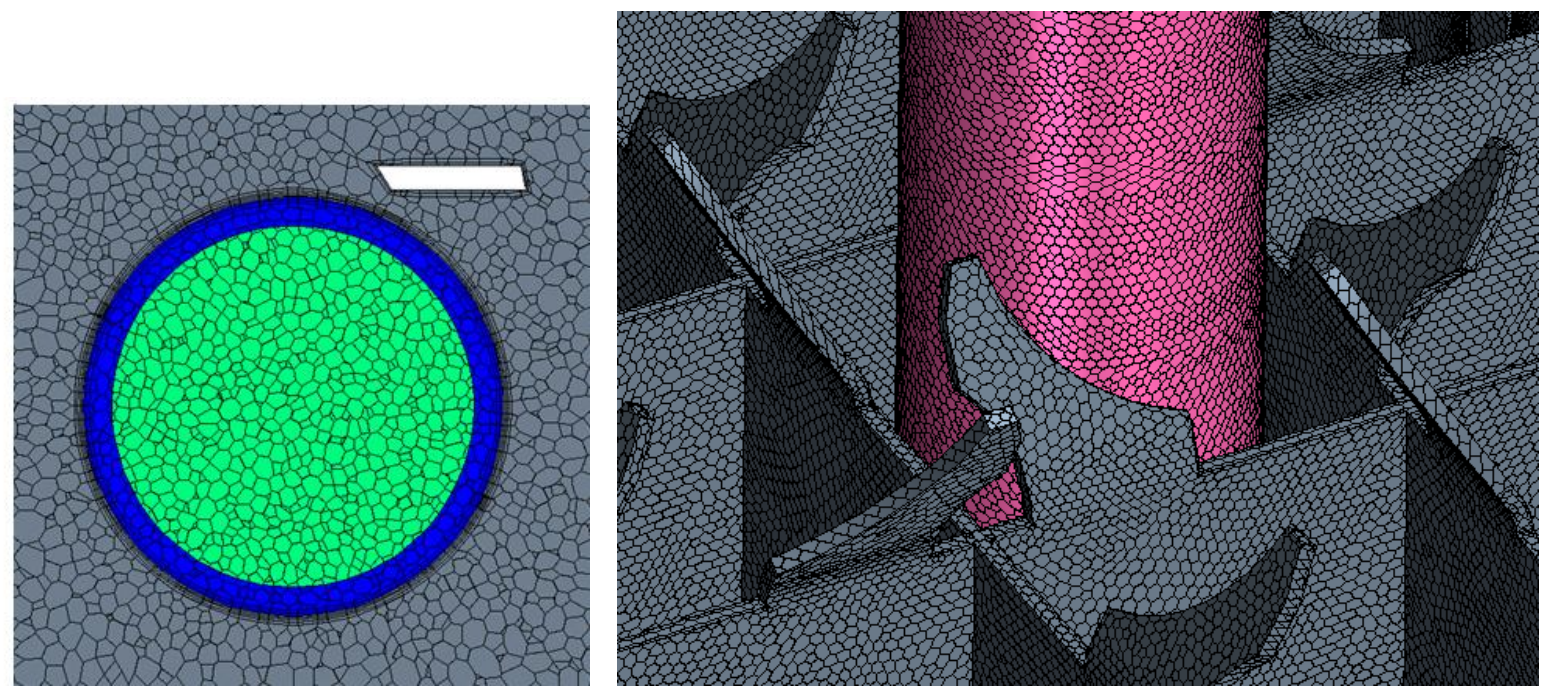

Fig. 4: Details of the CFD mesh - pin cross section (left) and mixing vanes (right). 


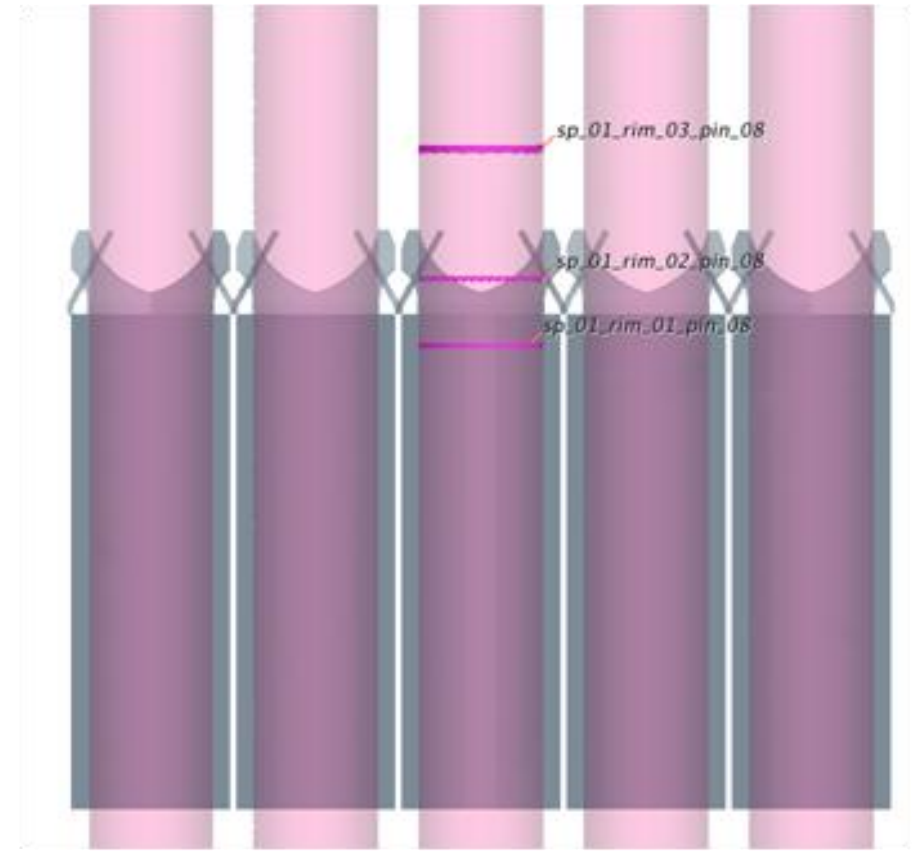

Fig. 5: Axial location near grid spacer \#5 for azimuthal distributions of Fig. 6. 

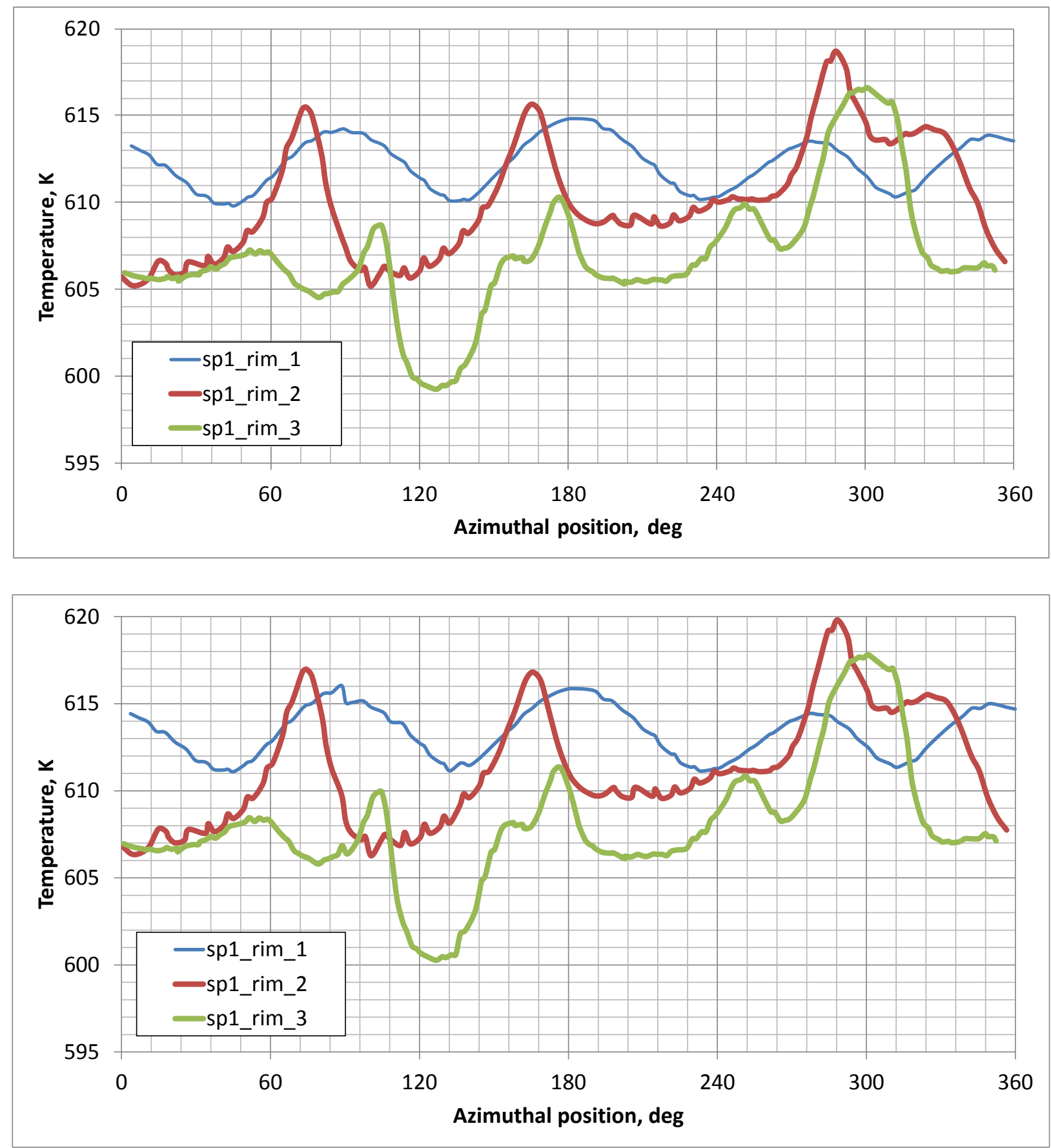

Fig. 6: Azimuthal distribution of cladding temperature for pin \#8, near grid spacer \#5, at 4 days (top) and 502 days (bottom) respectively. 

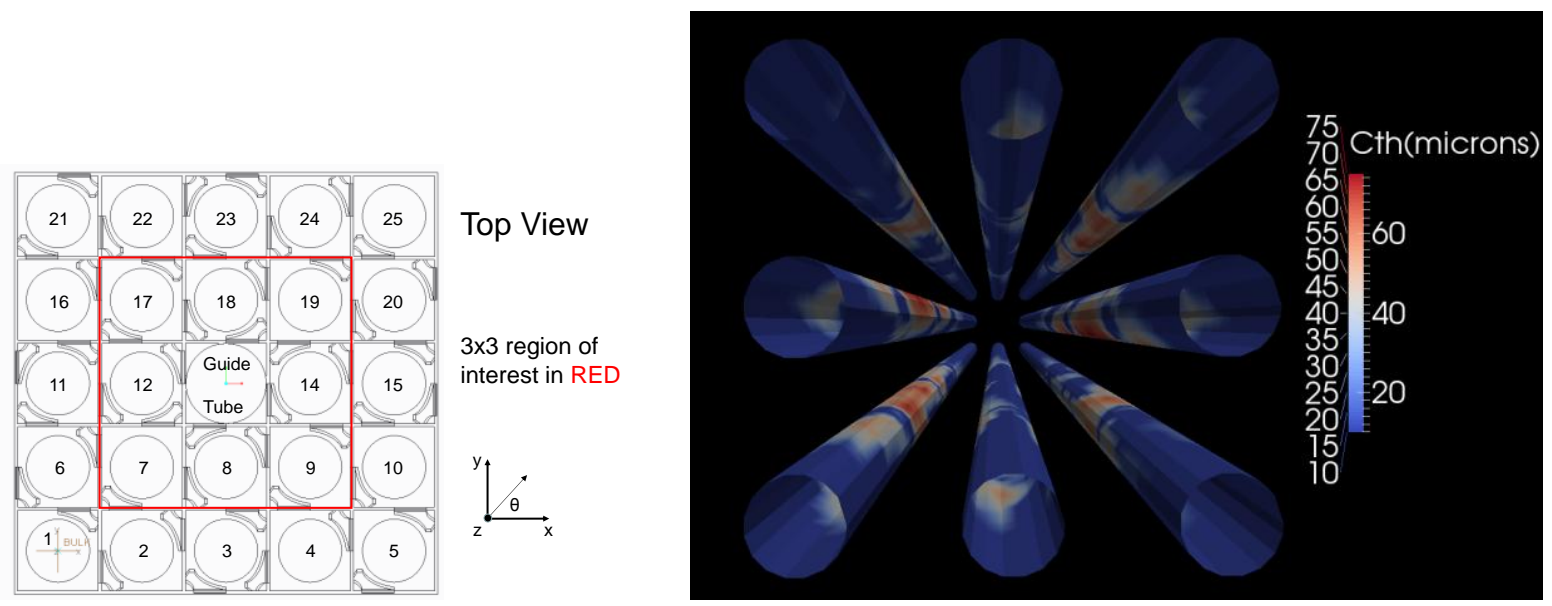

Fig. 7: 3D top view of $3 \times 3$ surrounding the center guide tube (not shown) at 502 days. The color scale indicates the CRUD thickness (in microns). 


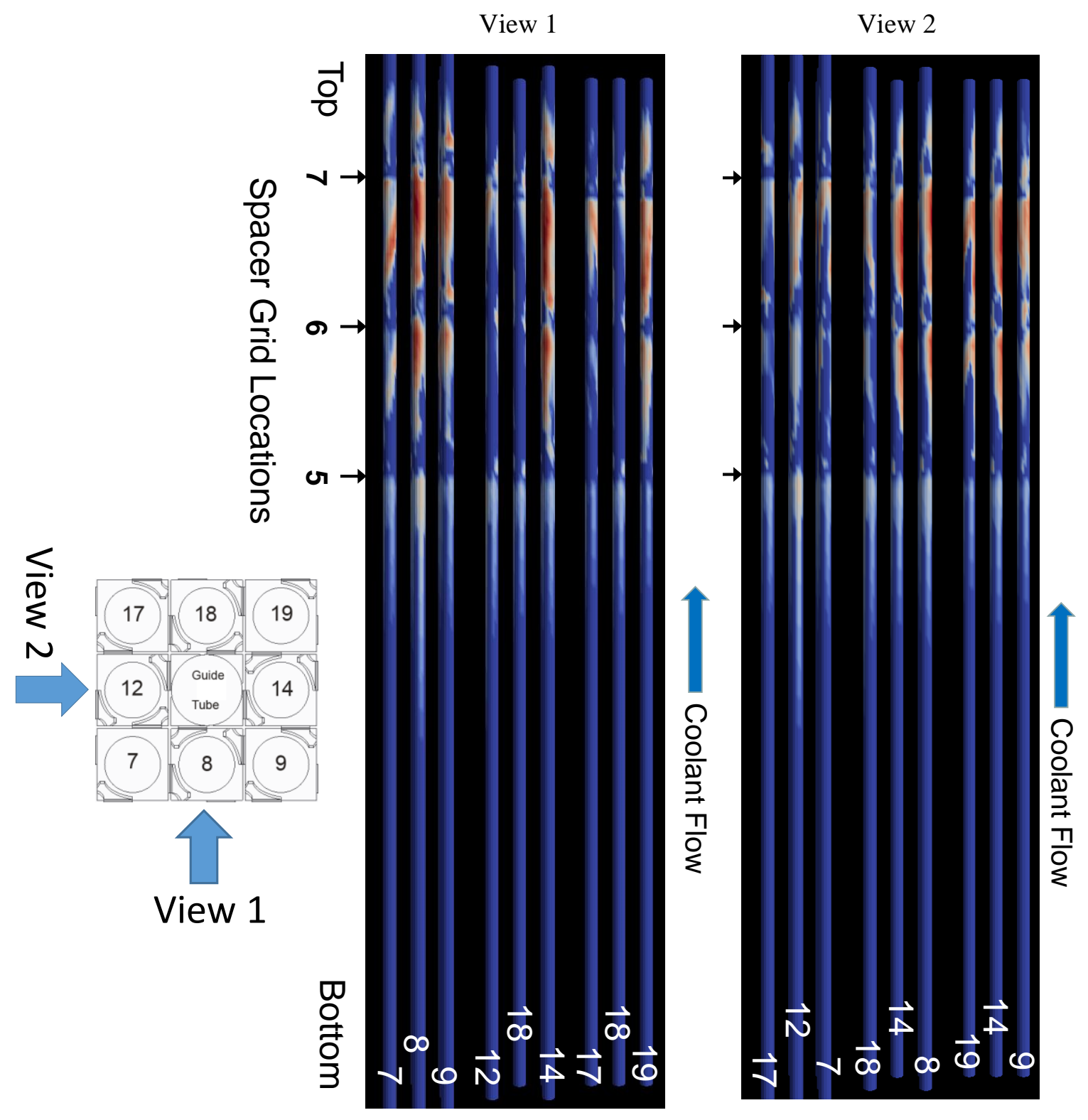

Fig. 8: $3 \times 3$ face views at 502 days. 


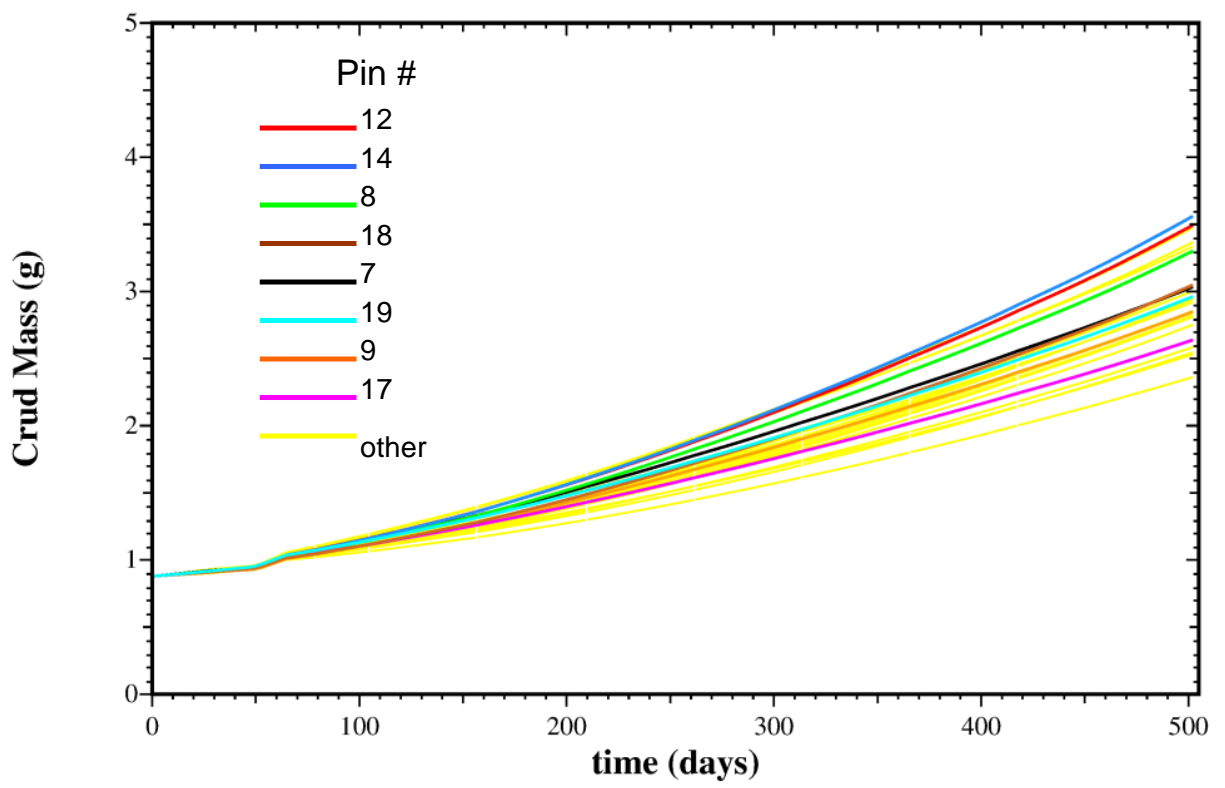

Fig. 9: Total CRUD mass on each pin vs time 

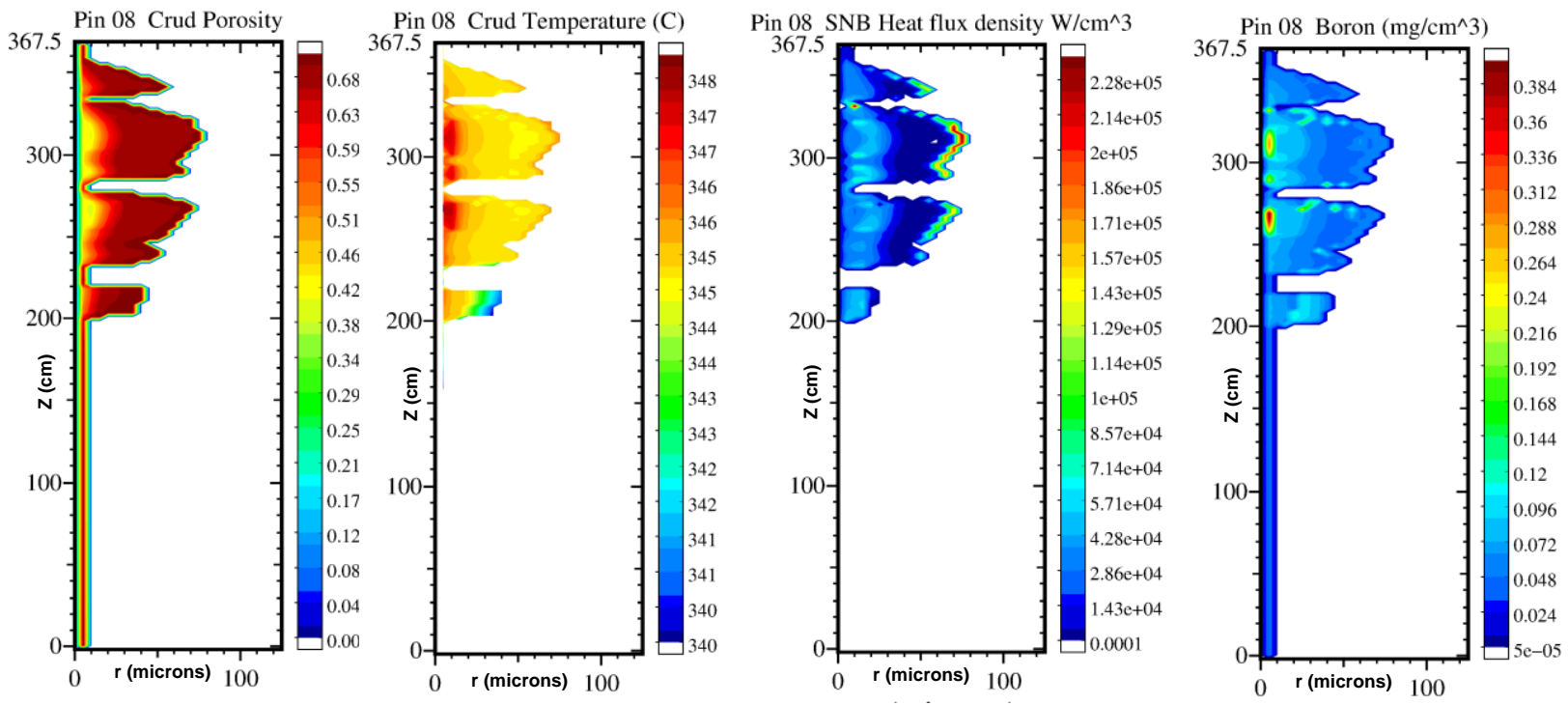

Fig. 10: 2D CRUD profiles at 502 days for pin \#8. 

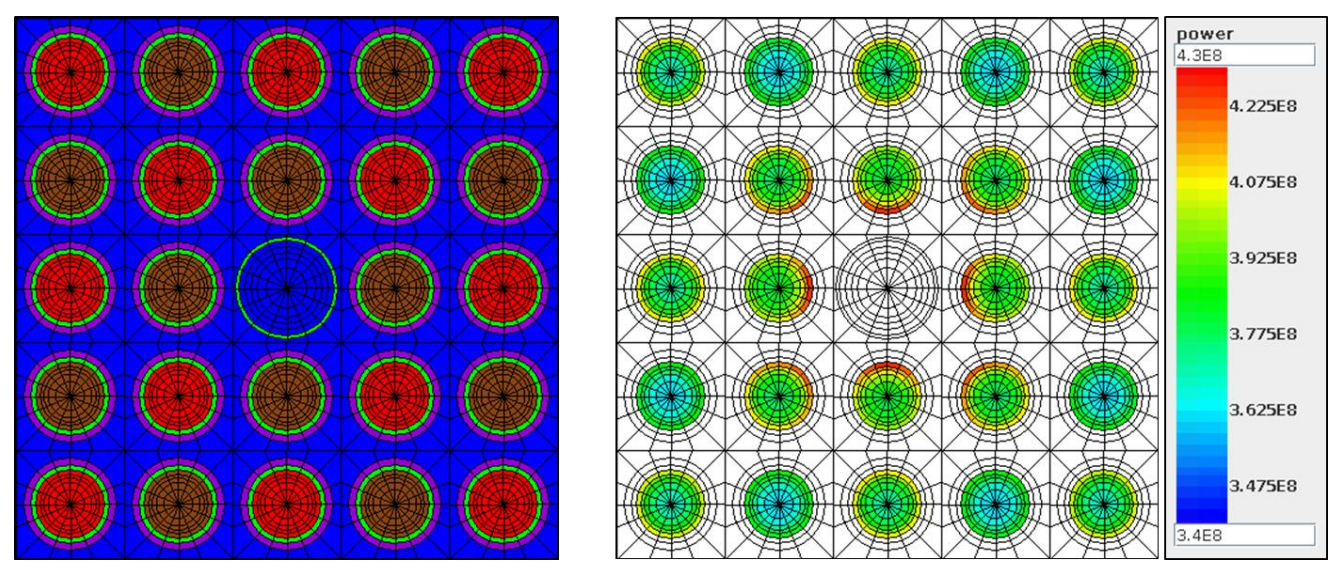

Fig. 11: DeCART 5x5 model showing material regions (left), and radial and azimuthal power distribution for mid-plane at 4 days (right). 

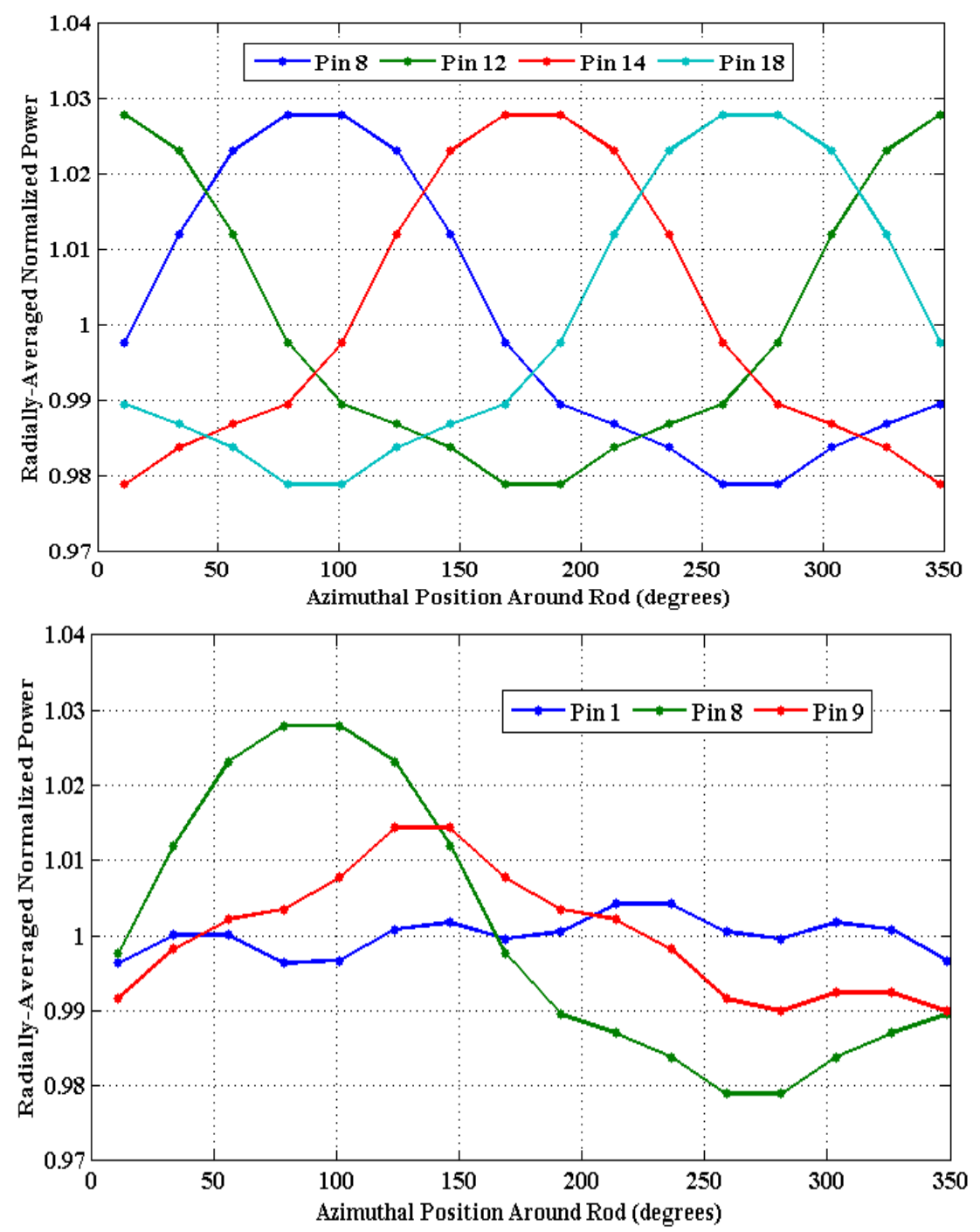

Fig. 12: Azimuthal power variation for different pins at 4 days in the cycle. 

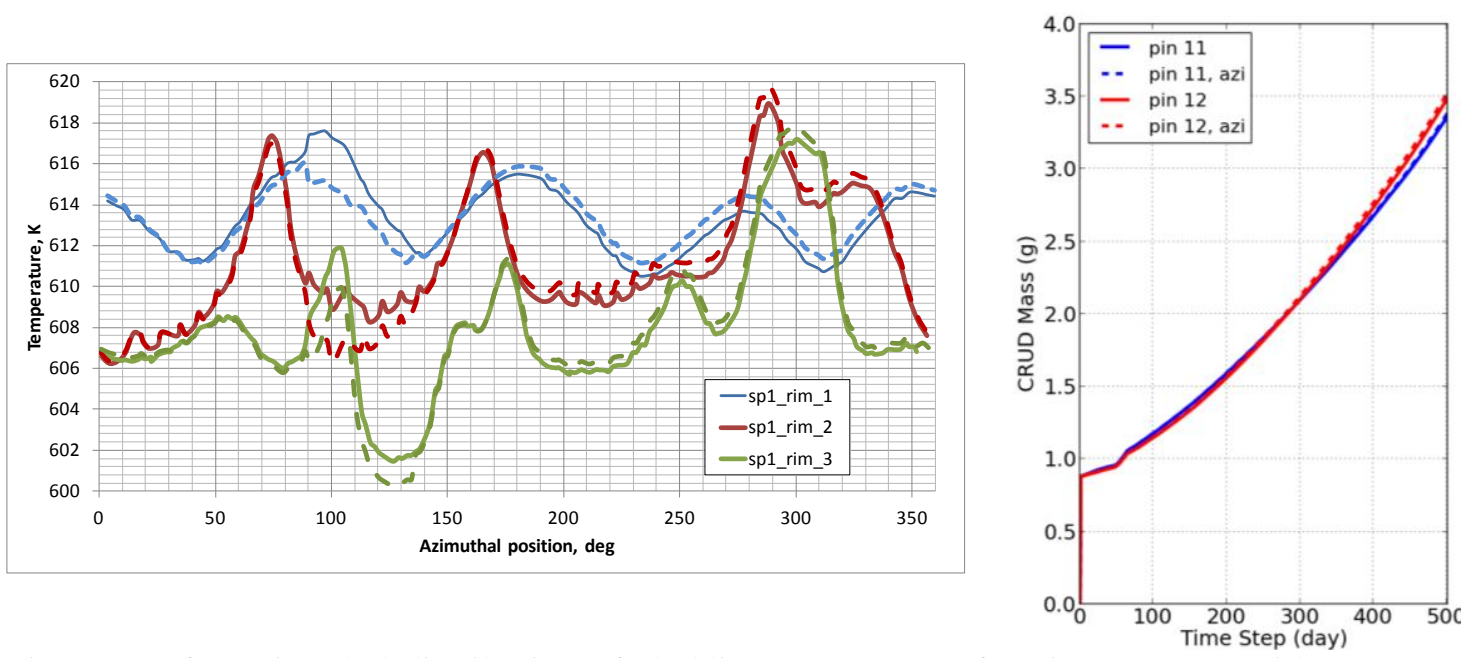

Fig. 13: Left - azimuthal distribution of cladding temperature for pin \#8, near grid spacer \#5, at 502 days (dashed lines for original ANC power, solid lines for azimuthally varying ANC power distribution. Right - integral CRUD mass comparison of ANC and azimuthally varying ANC power simulations for pin 11 and 12 . 

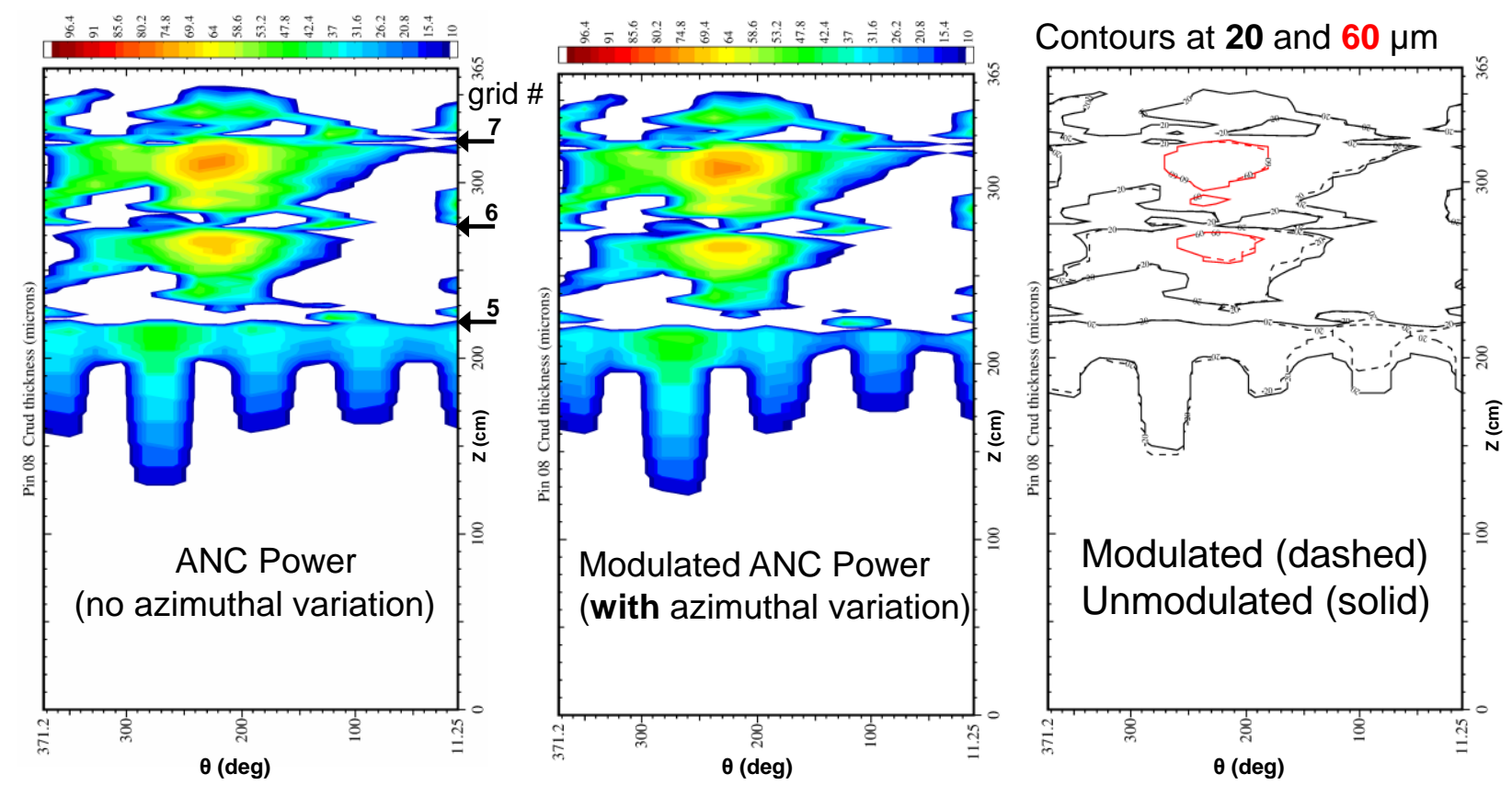

Fig. 14: 2D surface contour maps of the CRUD thickness (in microns at 502 days) for pin \#8 with and without azimuthal power variations. 


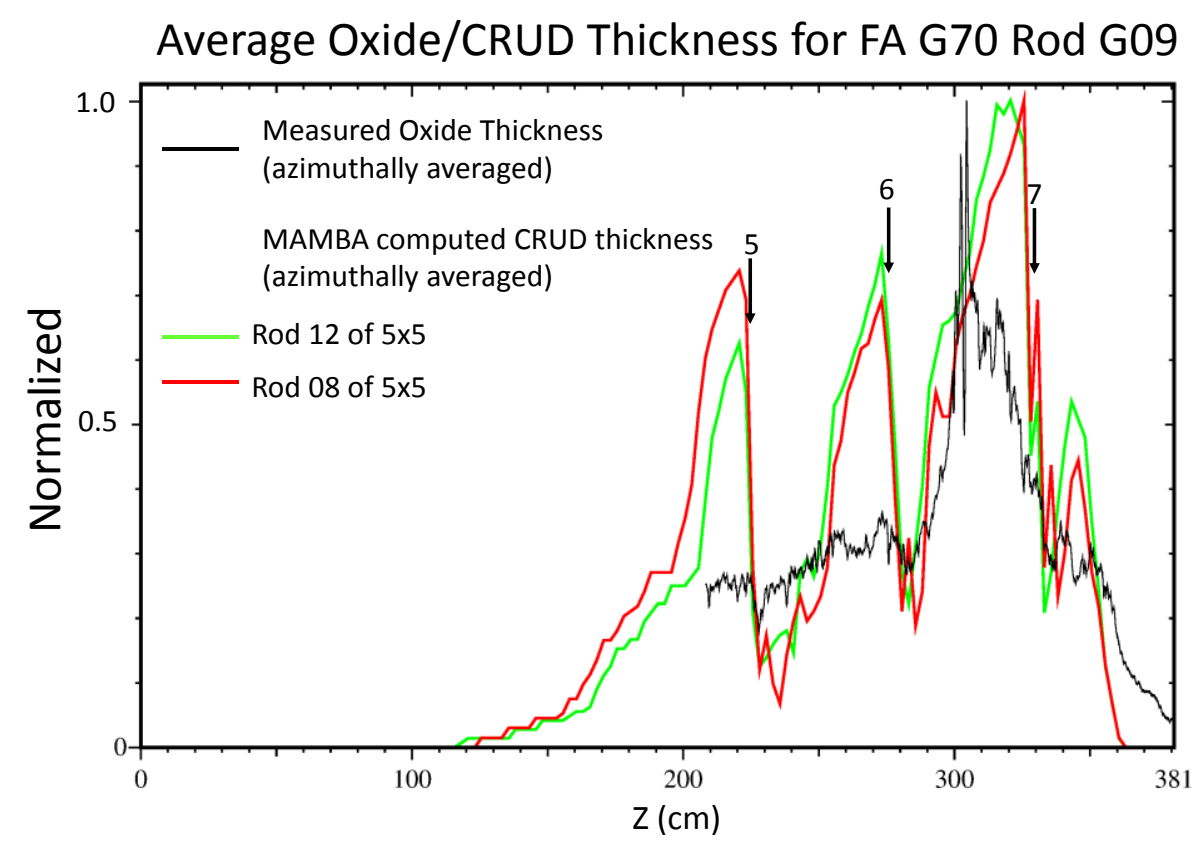

Normalized Oxide Thickness for FA G63 Rod G09

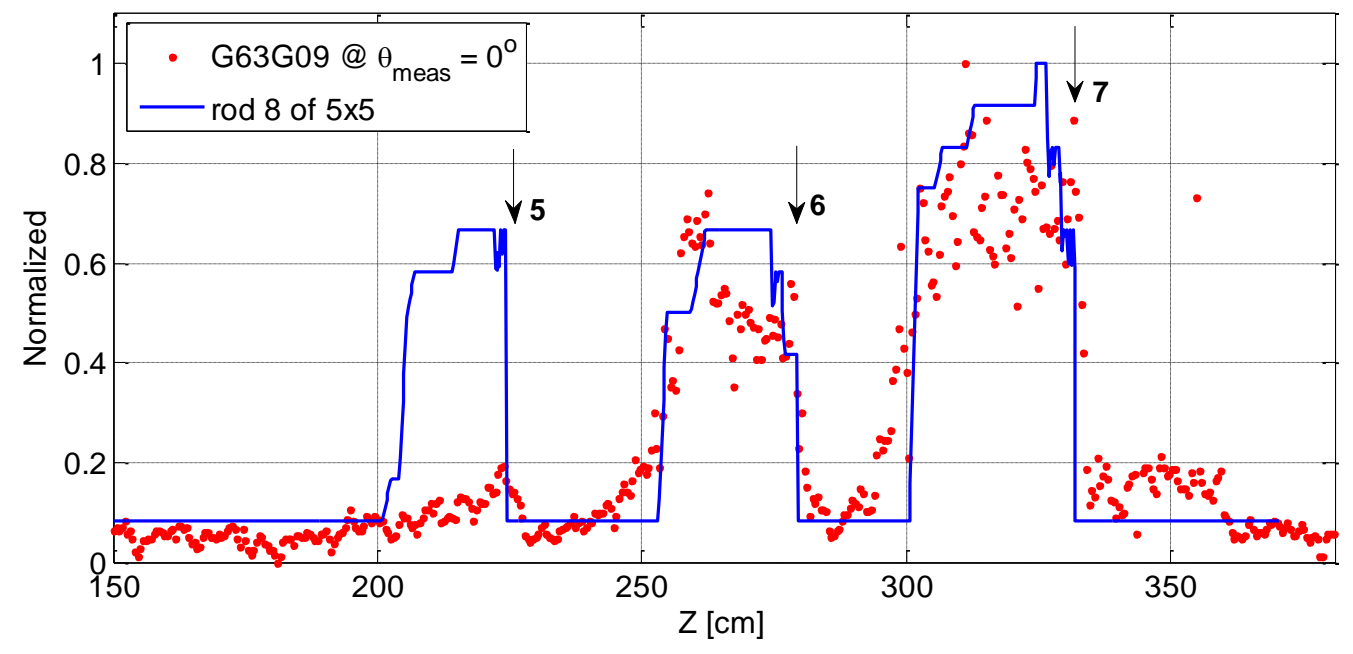

Fig. 15: Normalized azimuthally averaged oxide/CRUD thickness as function of fuel height 

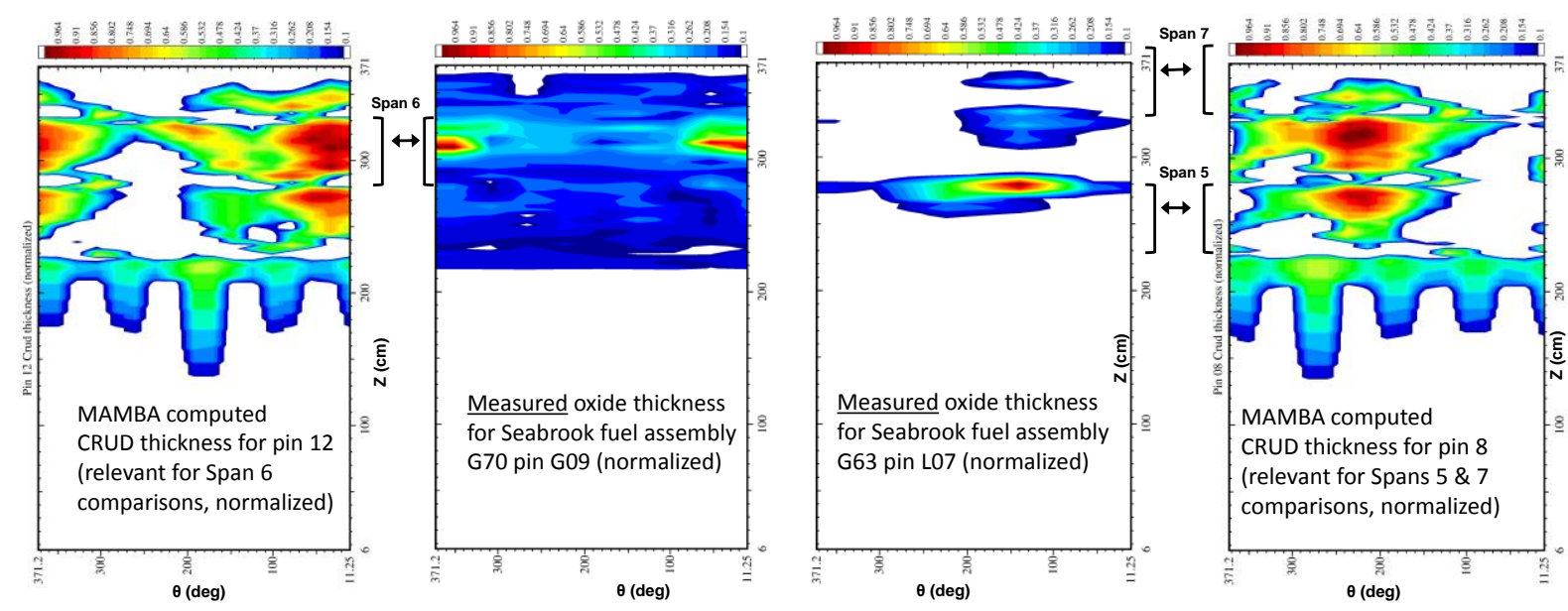

Fig. 16: 2D contour plots of the normalized computed CRUD thickness (MAMBA) and measured oxide thickness for Seabrook fuel assembly G70 rod G09 (left) and G63 rod L07 (right). 


\section{REFERENCES}

Cohen, P., "Heat and mass transfer for boiling in porous deposits with chimneys". AIChE Symposium Series, 70 (138), 1974.

Henshaw, J., et al., "A model of chemistry and thermal hydraulics in PWR fuel crud deposits", Journal of Nuclear Materials 353, 1-11, 2006.

Kendrick, B.K., Barber, J., "Initial validation and benchmark study of 3D MAMBA v2.0 against the WALT loop experiment and BOA v3.0”. CASL report MPOCRUD.P5.02, 2012.

Liu Y., et al, Pin Power Prediction in the Westinghouse Advanced Nodal Code, 1986, Trans. Am. Nuclear Soc., Vol. 53, pp 246.

OECD/NEA, "Report of the OECD/NEA-KAERI Rod Bundle CFD Benchmark Exercise", July 2013, NEA/CSNI/R(2013)5.

Petrov, V., Kendrick, B.K., Walter, D., Manera, A., "Impact of fluid-dynamic 3D spatial effects on the prediction of CRUD deposition in a 4x4 PWR sub-assembly", Proc. of NURETH-15, Pisa, Italy, 12 16 May 2013.

Secker, J., "AMA.CHALNG.07.Y1 Test Problem and Validation Data Package for CRUD", DOE/CASL deliverable, 2011.

Yan, J., Yun, P., Smith, L.D., Karoutas, Z.E., Jofrre, P., "PWR Fuel Sub-Channel Thermal Mixing CFD Model Development and Validation", Proc. of CFD4NRS-5, Zurich, Switzerland, 9 - 11 September 2014.

Walter, D., et al., "High-fidelity simulation of crud deposition on a fuel pin with grid spacers - a proofof-principle using the fully coupled MAMBA/DeCART/STAR-CCM+ code", Proc. of NURETH-15, Pisa, Italy, 12 - 16 May 2013. 